\title{
Recent HST Surveys of Planetary Nebulae in the Magellanic Clouds
}

\author{
Richard A. Shaw \\ NOAO, 950 N. Cherry Av., Tucson, AZ 85719 USA \\ Letizia Stanghellini, Max Mutchler \\ STScI, 3700 San Martin Dr., Baltimore, MD 21218 USA
}

Bruce Balick, Stacy Palen

Univ. Washington, Seattle, WA 21218 USA

Eva Villaver, J. Chris Blades

STScI, 3700 San Martin Dr., Baltimore, MD, 21218 USA

George Jacoby

WIYN Observatory, 950 N. Cherry Av., Tucson, AZ 85719 USA

Orsola De Marco

AMNH, Central Park West at 79th St., New York, NY 10024 USA

\section{Introduction}

The study of the formation and evolution of planetary nebulae (PNs) has been a subject of active investigation for several decades. In the past 15 years the relationship between nebular morphology and nebular/stellar evolution has been investigated in some detail. Although important insights have been gained, the connection between PN formation and even basic morphological features of the evolving nebula is far from clear. One of the most vexing problems to overcome is the difficulty of obtaining, at least for Galactic PNs where statistical distances must be adopted, reliable dimensions, ages, luminosities, and other physical quantities that are essential for understanding the evolutionary state of individual nebulae. Our emphasis of late has been to obtain high-resolution images of a large sample of PNs in the Magellanic Clouds, where uncertainties in the distances are minimal and selection effects due to, e. g., dust absorption in the Galactic plane do not apply.

\section{Survey Highlights}

We present broad-band images and slitless spectra from our recent surveys of Magellanic Cloud planetary nebulae (MCPNs) using the Space Telescope Imaging Spectrograph on-board HST. The spectrograms yield monochromatic images 
in up to 13 of the more prominent nebular emission lines, including $\mathrm{H} \beta$ and $\mathrm{H} \alpha$, [O III] $\lambda 4959,5007,[\mathrm{O}$ I] $\lambda \lambda 6300,6363$, [S III] $\lambda 6312,[\mathrm{~N} \mathrm{II}] \lambda \lambda 6548,6584, \mathrm{He}$ I $\lambda 6678$, and $[\mathrm{S} \mathrm{II}] \lambda \lambda 6717,6731$. The physical resolution is better than $0.03 \mathrm{pc}$, which is comparable to observations of Galactic PNs from the ground. These data on $27 \mathrm{PNs}$ in the SMC and $48 \mathrm{PNs}$ in the LMC (at this writing) nearly double the number of HST observations of MCPN from prior-generation $H S T$ surveys using a limited set of narrow-band filters. (Many of the early observations were compromised by the aberrated beam of $H S T$ prior to the installation of corrective optics.) Our survey will continue during the next year, with up to 100 new observations of LMC PNs.

These data are very rich in scientific content. Our ability to observe PN morphology over a factor of 30 in physical size and in a number of emission lines has allowed us to explore the variation of nebular morphology with size, age, ionization, density, optical depth, and metallicity. We have confirmed in MCPNs many of the relationships reported for Galactic PNe, such as the correlation of asymmetric morphological types with chemical enrichments (or depletions), and progenitor star Population type (Stanghellini, et al. 2000). The sample has also lead to refinements in the morphological classification scheme itself, including the recognition of the important bipolar-core type, which we have shown is a subclass of the bipolar type, rather than of the elliptical or round types (Shaw, et al. 2001). We are exploring further refinements to the morphological classification scheme, including methods to disambiguate the effects of projection, ionization, and mass distribution, which are important for comparisons with numerical models (Stanghellini, et al. 2002). Finally, we find significant differences between LMC and SMC samples in the incidence of morphological types, which when combined with the difference in mean chemical abundance of the host galaxies, may yield important insights into environmental or chemical influences on PN formation (Shaw, et al. 2001).

These samples of MCPNs are of substantial archival value, and we are endeavoring to provide public access to scientifically useful products that will enable additional research and new observations of MCPNs. All of the survey data obtained so far are in the public domain; data from all future observations (which will extend into 2002) will also be placed in the public domain immediately after they are obtained. Various value-added data products relating to our published papers, including processed FITS images, extracted spectra, and catalogs of nebular parameters, are offered on our MCPN Web site at http://archive.stsci.edu/hst/mcpn/.

\section{References}

Shaw, R. A., Stanghellini, L., Mutchler, M., Balick, B., \& Blades, J. C. 2001, ApJ, 548, 727

Stanghellini, L., Shaw, R. A., Balick, B., \& Blades, J. C. 2000, ApJ, 534, L167

Stanghellini, L., Shaw, R. A., Mutchler, M., Palen, S., Balick, B., \& Blades, J. C. 2002, ApJ, in preparation 